\title{
Comparison of the specificity of antibodies to VAR2CSA in Cameroonian multigravidae with and without placental malaria: a retrospective case-control study
}

Anna Babakhanyan ${ }^{1 *} \mathbb{D}$, Rui Fang ${ }^{1}$, Andrew Wey ${ }^{1}$, Ali Salanti ${ }^{3}$, Grace Sama², Canisia Efundem², Robert J. I. Leke ${ }^{2}$, John J. Chen", Rose G. F. Leke² and Diane W. Taylor ${ }^{1}$

\begin{abstract}
Background: Antibodies (Ab) to VAR2CSA prevent Plasmodium falciparum-infected erythrocytes from sequestrating in the placenta, i.e., prevent placental malaria (PM). The specificity of Ab to VAR2CSA associated with absence of PM is unknown. Accordingly, differences in the specificity of Ab to VAR2CSA were compared between multigravidae with and without PM who had Ab to VAR2CSA.

Methods: In a retrospective case-control study, plasma collected from Cameroonian multigravidae with $(n=96)$ and without $(n=324)$ PM were screened in 21 assays that measured antibody levels to full length VAR2CSA (FV2), individual VAR2CSA DBL domains, VAR2CSA domains from different genetic backgrounds (variants), as well as proportion of high avidity Ab to FV2.

Results: Multigravidae with and without PM had similar levels of Ab to FV2, the six VAR2CSA DBL domains and different variants, while the proportion of high avidity Ab to FV2 was significantly higher in women without PM at delivery $(p=0.0030)$ compared to women with PM. In a logistic regression model adjusted for gravidity and age, the percentage of high avidity Ab to FV2 was associated with reduced likelihood of PM in multigravidae. A 5 \% increase in proportion of high avidity Ab to FV2 was associated with a nearly $15 \%$ lower likelihood of PM.
\end{abstract}

Conclusion: Ab avidity to FV2 may be an important indicator of immunity to PM.

Keywords: Antibodies, Avidity, VAR2CSA, Placental malaria

\section{Background}

In pregnant women, Plasmodium falciparum-infected erythrocytes (IE) express the VAR2CSA malarial adhesin that mediates binding of IE to placental chondroitin sulfate A (CSA) [1, 2]. Pathology results from the accumulation of IE causing a condition called placental malaria (PM). PM endangers the health of pregnant women and the developing fetus [1]. Antibodies

\footnotetext{
*Correspondence: annab8@hawaii.edu

${ }^{1}$ Department of Tropical Medicine, Medical Microbiology and Pharmacology, John A Burns School of Medicine, University of Hawaii at Manoa, 651 Ilalo Street, BSB 320, Honolulu, HI 96813, USA

Full list of author information is available at the end of the article
}

(Ab) to VAR2CSA play an important role in protection from the adverse outcomes of PM in women living in malaria-endemic areas. Over successive pregnancies, women produce Ab to VAR2CSA that inhibit the binding of IE to CSA in vitro $[3,4]$, reduce maternal anaemia [5], lower placental parasitaemia at delivery [6, 7], increase the length of gestation [8], and improve infant birth weight [8]. VAR2CSA is a primary vaccine candidate for PM and is currently under clinical investigation [9]. Since full-length VAR2CSA is $350 \mathrm{kDa}$, researchers are exploring various sequences of VAR2CSA Duffybinding-like (DBL) domains to develop a vaccine, including the N-terminal ID1-ID2a sequence that contains the CSA-minimal binding site [10], DBL1-2 and 
DBL3x [11] and DBL1x-DBL3x [12] and C-terminal domains $4(\mathrm{DBL} 4 \varepsilon)$ and 5 (DBL5e) [13]. In addition to the selection of VAR2CSA DBL domain(s) for inclusion in a vaccine, other Ab properties may affect vaccine efficacy, such as the ability to inhibit IE binding to CSA [4, $6,10-12]$, ability to mediate opsonic phagocytosis [5, 14-17], a broad Ab repertoire to multiple VAR2CSA DBL domains [18] and high avidity Ab to the full-length VAR2CSA (FV2) early in pregnancy [19]. A vaccination strategy that could elicit long-term immunity with cross protection against different $P$. falciparum strains will be of great value and have an impact on global public health. Long-term humoral immunity is produced in germinal centers [20], where antigen-stimulated B cells undergo clonal expansion, and immunoglobulin classswitching, somatic hypermutation and clonal selection, leading to affinity maturation of $\mathrm{Ab}[21,22]$. As the $\mathrm{B}$ cell response matures, $\mathrm{Ab}$ levels increase, more epitopes are recognized and $\mathrm{Ab}$ quantity and quality increase. The pattern of $\mathrm{Ab}$ maturation and its role in $\mathrm{Ab}$ effector responses against PM in low-transmission areas remain unexplored.

This study examined specificity, including quantity and quality of the immune response to VAR2CSA, among Cameroonian multigravid women living in malaria lowtransmission areas. Archival samples used were collected before implementation of intermittent preventive treatment (IPT) with sulfadoxine-pyrimethamine and insecticide-treated bed nets; thus, women developed significant levels of immunity to PM. However, a proportion of multigravidae, even after three pregnancies, had PM at delivery. Therefore, it is plausible that in malaria lowtransmission settings multigravidae continue to acquire protective immune responses to PM even after the second pregnancy. Hence, the objective of this study was to identify immune responses to VAR2CSA that were present in women who were PM- that were absent in multigravidae with PM. Plasma samples from 420 multigravid women who were PM+ $(\mathrm{n}=96)$ and $\mathrm{PM}-(\mathrm{n}=324)$ were screened in 21 serological assays that measured IgG to full-length VAR2CSA (FV2); the six VAR2CSA DBL domains, including variants from different genetic background, proportion of Ab with high avidity to FV2 (i.e., per cent of $\mathrm{Ab}$ that remained bound to FV2 in the presence of $3 \mathrm{M} \mathrm{NH}_{4} \mathrm{SCN}$ ), total number of DBL domains recognized, as well as $\mathrm{Ab}$ levels to non-pregnancy specific malarial antigens (MSP-1, MSP-2, AMA-1, RESA, CSP). Identifying fine immunological differences between women with PM and those without could expedite VAR2CSA-based vaccine development, a vaccine that could protect an estimated 85 million women and their fetuses worldwide from the severe effects of malaria [23].

\section{Methods}

\section{Ethical consideration}

The archival, coded samples used in the current study were exempt from human subject research by the Committee on Human Studies, University of Hawaii, Manoa (CHS\#19912). The original studies were conducted according to the Helsinki Declaration principles and approved by the National Ethics Committee, Cameroon and the Institutional Review Board at Georgetown University. All participants gave written informed consent to use their blood samples to measure Ab to malaria.

\section{Study design and plasma samples}

In this retrospective case-control study, archival plasma samples from a previous large cross-sectional study conducted between 1996 and 2001 [24, 25] were used. All the samples were collected at delivery from Cameroonian women living in Yaoundé. Yaoundé, the capital of Cameroon, is a malaria-endemic area, where entomological inoculation rates are estimated to be 13 infectious bites per person per year [26, 27]. Since the samples were collected before implementation of IPT and long-lasting insecticide treated bed nets, all of the women were likely to have become infected several times during pregnancy. Although the human immunodeficiency virus (HIV) status of the women is unknown, the prevalence of HIV among pregnant women attending antenatal-care clinics in 2001 in urban areas in Cameroon is estimated to be $4.0-13.6 \%$ [28], making it unlikely that HIV had a major effect on the results. Since $P$. falciparum transmission is low in Yaounde, plasma samples were screened for Ab to FV2 (FCR3 strain) (see cut-off based on Cameroonian males in Additional file 1), to confirm that the women had become infected and produced Ab to FV2. Only plasma samples from women who were seropositive to FV2 FCR3 were further studied. The following inclusion criteria were used: multigravidae ( $\geq 3$ pregnancies), 18 years or older, singleton live deliveries with babies that were $>28$ weeks of gestation, and had Ab to FV2. All multigravidae who fit the inclusion criteria and had PM were included in the study $(\mathrm{n}=96)$. These multigravidae should have acquired immunity to PM during their previous $\geq 2$ pregnancies, however, since they had PM it is likely they had not developed a complete protective immune response. For comparison, about three times the number $(\mathrm{n}=324)$ of PM-negative multigravidae that met the inclusion criteria were randomly selected. Archival plasma samples from American pregnant women ( $n=42$ ) were used to establish the cut-off for seropositivity to non-pregnancy-specific malaria antigens. Twenty Cameroonian male plasma samples were used to establish cut-off for seropositivity to VAR2CSA antigens (cut-off for FV2 FCR3 was 2,326 median fluorescence 
intensity (MFI), cut-off for other antigens is presented in Additional file 1). Pre-term deliveries were defined as less than 37 weeks of gestation; low birth weight was defined as less than $2500 \mathrm{~g}$.

\section{Diagnosis of placental malaria and anaemia}

Thick and thin blood smears were prepared using maternal peripheral and placental intervillous space blood, and impression smears were made from biopsies of placental tissues. Slides were stained with Diff-Quick (Polysciences, Warrington, PA, USA, Cat no: 24606-250) and read by two microscopists to determine parasitaemia. Placental biopsies were also fixed in buffered formalin, embedded, stained with haemotoxylin-eosin, and examined for parasites. A woman was considered PM-positive $(\mathrm{PM}+)$ if IE were detected in blood smears of intervillous space blood, impression smears of villous tissue, or histological sections of the placenta [18]. Maternal peripheral blood was used to determine the haematocrit/packed cell volume (PCV). Using the definition of the World Health Organization, a woman was considered anaemic if the haematocrit/PCV was less than $30 \%$ [25].

\section{Recombinant proteins}

The panel of recombinant proteins of different regions of VAR2CSA have been described previously [18], and included: full-length VAR2CSA (FV2), DBL1 + 2, DBL 2, DBL 3, DBL 4, DBL 5, DBL 6 of the FCR3 strain expressed in Baculovirus-transfected insect cells at the University of Copenhagen, Denmark; DBL1, DBL3, DBL4, DBL5, and DBL6 for the 7G8, 3D7 and IT4 strains expressed in Pichia pastoris from J D Smith and colleagues (Seattle Biomedical Research Institute, USA) [29], and DBL 3 (A4 expressed in Escherichia coli provided by K Singh (NIAID, NIH, Bethesda, MD, USA). The FCR3 and IT4 parasite lines are isogenic due to historical contamination [30] and therefore designated in the manuscript as FCR3/IT4. In addition, non-pregnancy-associated malarial antigens were used, including recombinant AMA-1 $3 \mathrm{D} 7$ (expressed in yeast), MSP-1 ${ }_{42} 3 \mathrm{D} 7$ (Escherichia coli), and MSP-2 3D7 were provided by C Long, Malaria Vaccine Development Branch (MVDB), National Institute of Allergy and Infectious Disease, National Institutes of Health, Rockville, MD, USA. Synthetic peptides containing $B$ cell epitopes from CSP and RESA were synthesized by AnaSpec, Inc. (San Jose, CA, USA) and reported previously in detail [31].

\section{Coupling of recombinant proteins to SeroMAP microspheres}

The method used for coupling was reported previously [31]. Proteins were coupled at optimal concentrations to 1 million microspheres: DBL1-DBL6 at $1 \mu \mathrm{g}, \mathrm{FV} 2$ at $3 \mu \mathrm{g}$,
AMA-1 3D7 and MSP-1 3D7 at $1 \mu \mathrm{g}, \mathrm{RESA}$ at $40 \mu \mathrm{g}$, CSP at $15 \mu \mathrm{g}$ and MSP-2 3D7 at $0.2 \mu \mathrm{g}$.

\section{Measuring IgG using a multi-analyte platform assay}

The multi-analyte platform (MAP) assay was performed as previously described $[18,19,31]$. Briefly, $50 \mu \mathrm{l}$ of antigen-coupled microspheres (2000 microspheres/test) were incubated with $50 \mu \mathrm{l}$ of a 1:100 dilution of plasma in phosphate buffered saline (PBS) containing $1 \%$ bovine serum albumin (BSA) in pre-wetted filter plates (96-well Multiscreen BV; Millipore, Billerica, MA, USA), for $1 \mathrm{~h}$ at $25{ }^{\circ} \mathrm{C}$ on a rotating shaker at $500 \mathrm{rpm}$ (Microplate Shaker, Lab-line, Melrose Park, IL, USA). Microspheres were washed twice with PBS-0.05\% Tween20 and once with PBS-1 \% BSA. Then, $100 \mu \mathrm{l}$ of secondary Ab (R-phycoerythrin-conjugated, Affini Pure $\mathrm{F}\left(\mathrm{ab}^{\prime}\right)_{2}$ fragment, Goat anti-human IgG Fc fragment specific, Jackson Immunoresearch, West Grove, PA, USA, Cat no. 109116-170) diluted to $2 \mu \mathrm{g} / \mathrm{ml}$ in PBS-1 \% BSA was added to each well and incubated as above in the dark for $1 \mathrm{~h}$. Wells were washed as described above, microspheres were re-suspended in $100 \mu \mathrm{l}$ PBS-1 \% BSA and the microsphere suspension was analysed using a Liquichip M100 reader (Qiagen, Valencia, CA, USA). The reader was programmed to read a minimum 100 beads per spectral address, DD Gate 7500-15,000 and 35-s timeout. The results were expressed as MFI. Positive and negative controls were included on each plate consisting of three different pools of plasma from eight Cameroonian multigravidae with high Ab levels to VAR2CSA and pools of plasma from 40 Americans who had never travelled to malaria-endemic areas.

\section{IgG subclass determination to the FV2}

For IgG subclass assay, each plasma sample was tested in quadruplicates. After initial incubation of FV2coupled microspheres with plasma and washing as described above, microspheres were incubated with 100 $\mu \mathrm{L}$ mouse anti-human $\mathrm{Ab}$ to detect specific IgG subclasses: (a) mouse anti-human IgG1 (Molecular Probes, Ref. A10630) 1:1000 dilution, (b) mouse anti-human IgG2 (Sigma, ascites HP6014 I5635) 1:1000 dilution, (c) mouse anti-human IgG3 (Sigma, clone HP6050 I7260) 1:2000 dilution, and, (d) mouse anti-human IgG4 (Sigma HP6023 I9888) 1:1000 dilution. Plates were incubated for $1 \mathrm{~h}$, at $25^{\circ} \mathrm{C}$ on the shaker. After washing as described above, microspheres were resuspended in $100 \mu \mathrm{l}$ of R-phycoerythrin-conjugated donkey anti-mouse IgG $\mathrm{H}+\mathrm{L}$ (Jackson 715-116-150) at 1:250 dilution. Plates were incubated for $1 \mathrm{~h}$, at $25{ }^{\circ} \mathrm{C}$ on a shaker, washed as before and resuspended in $100 \mu \mathrm{l}$ PBS-1 \% BSA; $85 \mu \mathrm{l}$ of the microsphere suspension was analysed using a Liquichip M100 reader (Qiagen, Valencia, CA, USA). To test 
the quality of mouse-anti-human $\mathrm{Ab}$, beads coupled to human monoclonal IgG1k, human monoclonal IgG2k, human monoclonal IgG3 $\lambda$ and human monoclonal IgG4 $\lambda$ were used. These beads were assayed as described above. Each sub-class was highly specific with no significant cross-reactivity with other IgG sub-classes (Additional file 2).

\section{Avidity to the FV2}

The avidity assay was performed as previously described [19]. Briefly, plasma was diluted 1:300, 1:1000 and 1:3000 in PBS-1 \% BSA and $50 \mu \mathrm{l}$ of diluted plasma was added to six wells (each dilution in duplicate) containing $50 \mu \mathrm{l}$ of FV2-coupled microspheres (2000 microspheres/test) and incubated for $1 \mathrm{~h}$ on a shaker at $25^{\circ} \mathrm{C}$. After incubation, $100 \mu \mathrm{l}$ of $3 \mathrm{M} \mathrm{NH}_{4} \mathrm{SCN}$ in PBS-\% BSA was added to half of the wells and $100 \mu \mathrm{l}$ of PBS-1 \% BSA was added to the other half (matching control wells for each dilution). After $30 \mathrm{~min}$ of incubation, the wells were washed as above and incubated with secondary Ab. Secondary $\mathrm{Ab}$ was washed as above and beads resuspended in $100 \mu \mathrm{l} \mathrm{PBS}-1 \%$ BSA and analysed by MicroChip 100 as described in the MAP assay. Proportion Ab that remained bound (Ab avidity) in the presence of $3 \mathrm{M}$ $\mathrm{NH}_{4} \mathrm{SCN}$ were determined for each dilution by the following formula: (MFI obtained from wells incubated with salt)/(MFI obtained from corresponding control wells) $\times 100 \%$, and the average was taken across three dilutions. Positive control consisting of pool of plasma from multigravida Cameroonian women and negative control consisting of pool of American plasma were included on each plate. Based on data from a previous study [19] when $\geq 35 \%$ of $\mathrm{Ab}$ remained bound in the presence of $3 \mathrm{M} \mathrm{NH}_{4} \mathrm{SCN}$, women were classified as having high avidity $\mathrm{Ab}$.

\section{Determining the breadth of the VAR2CSA antibody response}

The panel included 15 recombinant variants from three different strains (i.e., 3D7, FCR3, 7G8) that represent the six VAR2CSA DBL domains. A woman was considered to be Ab-positive for each of the 15 antigens if her plasma had a MFI greater than the mean MFI plus two standard deviations obtained with 20 plasma samples from Cameroonian males (unique cut-off was used for each antigen, see Additional file 1). In determining the breadth of the $\mathrm{Ab}$ response (repertoire), the total number of variants a woman recognized was determined, giving a score of $0-15$ variants (note: DBL1 +2 was excluded from this analysis, since it encompasses two DBL domains). A woman was considered Ab-positive for a particular domain, if she had $\mathrm{Ab}$ to one or more variant of that domain, i.e., giving a breadth of DBL domains of 0-6. Mean Ab levels plus two standard deviations of American controls was used as a cut-off for seropositivity to non-pregnancy-specific malarial antigens.

\section{Statistical analysis}

Demographic, clinical and assay variables were summarized with means and standard deviations for the continuous variables, and frequencies and percentages for categorical variables. $\mathrm{PM}+$ and $\mathrm{PM}-$ women were compared using both parametric and non-parametric approaches. As results were consistent, only parametric results (e.g., two sample t-tests with unequal variances for continuous variables and Chi square tests with a continuity adjustment, or Fisher's exact, when applicable, for categorical variables) were reported. Finally, logistic regression models were developed to assess the association between PM status and each $\mathrm{Ab}$ response with and without adjustment for demographic and/or clinical variables. For ease of result interpretation, the assay data (MFI) were rescaled by interquartile range (IQR, the difference between 75th and 25th percentiles). All statistical analyses were performed using SAS 9.4 and GraphPad Prism; p value less than 0.05 was regarded as statistically significant.

\section{Results}

\section{Characteristics of study population}

The study population is described in Table 1 . $\mathrm{PM}+$ women were on average of 2 years younger $(\mathrm{p}=0.0012) ; \mathrm{PM}+$ women on average had $4.5 \pm 1.6$ pregnancies, while PM-negative women had $5.2 \pm 2.0$ pregnancies $(p=0.0002)$. Seventy-one per cent $(68 / 96)$ of $\mathrm{PM}+$ women were also peripheral blood smear positive. Mean placental parasitaemia determined by impression smears was $3.5 \%$ (7.6 \%). Haematocrit levels were significantly lower in $\mathrm{PM}+$ women $(<0.0001)$, resulting in a higher proportion of $\mathrm{PM}+$ who were anaemic at delivery $(\mathrm{p}=0.0055)$. No significant differences were observed between PM+ and PM- multigravidae in terms of prevalence of pre-term deliveries or neonate birth weight.

\section{Differences in immune responses to VAR2CSA \\ between multigravidae with and without PM}

Multigravidae with PM and without PM had similar Ab levels to DBL1 $+2(\mathrm{p}=0.37)$, DBL2 $(\mathrm{p}=0.53)$, DBL3 $(\mathrm{p}=0.32)$, DBL4 $(\mathrm{p}=0.78)$, DBL5 $(\mathrm{p}=0.54)$, DBL6 $(\mathrm{p}=0.54)$, and FV2 $(\mathrm{p}=0.76)$ from the FCR3 strain and on average recognized similar number of domains $(4.2 \pm 1.3)$ and variants $(9.3 \pm 3.7)$ (Table 2). Similar data were obtained for the other strain variants (Table 2). However, PM+ women had significantly lower avidity 
Table 1 Characteristics of pregnant women and their neonates

\begin{tabular}{|c|c|c|c|}
\hline Characteristic $^{a}$ & $\begin{array}{l}\text { PM positive } \\
(n=96)\end{array}$ & $\begin{array}{l}\text { PM negative } \\
(n=324)\end{array}$ & p value \\
\hline Age in years (mean $\pm S D$ ) & $27.6 \pm 4.6$ & $29.4 \pm 5.6$ & 0.0012 \\
\hline Gravidity (mean \pm SD) & $4.5 \pm 1.6$ & $5.2 \pm 2.0$ & 0.0002 \\
\hline Prevalence of women with peripheral malaria by blood smears, n (\%) & $68(70.8 \%)$ & $5(1.5 \%)$ & $<0.0001$ \\
\hline Peripheral malaria parasitaemia among all women, parasites/ $\mu \mathrm{L}$ (mean \pm SD) & $0.2 \pm 0.6$ & $0.0 \pm 0.02$ & 0.0011 \\
\hline Peripheral malaria parasitaemia among blood smear positive women parasites/ $\mu \mathrm{L}$ (mean $\pm \mathrm{SD}$ ) & $0.3 \pm 0.7$ & $0.1 \pm 0.5$ & 0.12 \\
\hline Prevalence of women with placental parasitaemia, n (\%) & $96(100 \%)$ & $0(0 \%)$ & \\
\hline Placental parasitaemia by impression smears, (\%) (mean \pm SD) & $3.5 \pm 7.6$ & $0 \pm 0$ & \\
\hline Pre-term deliveries, n (\%) & $16(16.7 \%)$ & $63(19.4 \%)$ & 0.59 \\
\hline Length of pregnancy in weeks (mean \pm SD) & $39.1 \pm 2.7$ & $39.1 \pm 3.0$ & 0.95 \\
\hline Full-term deliveries & $39.9 \pm 1.7$ & $40.3 \pm 1.5$ & 0.12 \\
\hline Pre-term deliveries & $34.3 \pm 1.7$ & $34.0 \pm 2.4$ & 0.50 \\
\hline Intra-uterine growth restriction, n (\%) & $2(2.1 \%)$ & $9(2.8 \%)$ & 0.99 \\
\hline Maternal anaemia at delivery, n (\%) & $24(25.0 \%)$ & $39(12.0 \%)$ & 0.0055 \\
\hline Haematocrit in \% (mean \pm SD) & $32.0 \pm 5.0$ & $34.7 \pm 5.1$ & $<0.0001$ \\
\hline Low birth weight babies, n (\%) & $11(11.5 \%)$ & $30(9.3 \%)$ & 0.68 \\
\hline Among full-term deliveries & $2(2.5 \%)$ & $8(3.2 \%)$ & 1.00 \\
\hline Among pre-term deliveries & $8(53.3 \%)$ & $20(33.9 \%)$ & 0.30 \\
\hline Neonate weight in grams (mean \pm SD) & $3139 \pm 600$ & $3257 \pm 611$ & 0.10 \\
\hline Full-term deliveries & $3303 \pm 433$ & $3393 \pm 478$ & 0.13 \\
\hline Pre-term deliveries & $2337 \pm 667$ & $2681 \pm 739$ & 0.10 \\
\hline
\end{tabular}

${ }^{a}$ For the univariate analyses, two-sample t tests for continuous variables and Chi square or Fisher's exact tests for categorical variables were used to compare women in $\mathrm{PM}+$ and $\mathrm{PM}$ -

$p$ values that were less than 0.05 were designated in italics

to FV2 ( $\mathrm{p}=0.0030)$ compared to multigravidae without PM, although the prevalence of women with high $\mathrm{Ab}$ avidity ( $\geq 35 \%$ ) was not statistically significant between the two groups (Table 2). Ab to non-pregnancy-specific malarial antigens were associated with infection: AMA-1 $(\mathrm{p}<0.0001)$, MSP1 $(\mathrm{p}=0.035)$, and MSP2 $(\mathrm{p}=0.0028)$.

To test whether VAR2CSA IgG sub-class distribution differed between women with and without PM, VAR2CSA IgG1, IgG2, IgG3, and IgG4 levels were measured in a sub-set of randomly selected $20 \mathrm{PM}+$ and 20 $\mathrm{PM}-$ women. Both $\mathrm{PM}+$ and PM- multigravidae predominantly had only IgG1 to FV2 (Additional file 3).

Of note, seven out of twenty Cameroonian males were seropositive for VAR2CSA domains (data not shown). One male was seropositive for DBL1, DBL4, DBL5, DBL6 and FV2 FCR3, another male for DBL1 + 2, DBL3, DBL4, remaining five males were seropositive for one or two domains only. There was no single domain that was consistently cross-reactive in majority or all males, rather domain(s) to which Cameroonian males were seropositive to varied between individuals. Seven males recognized total of 19 domain variants, compared to women's average for that particular domain: 13/19 had low Ab levels and additional individual had low Ab levels to FV2 FCR3, 2/19 had medium Ab levels and 4/19 had high Ab levels.

\section{Association between Ab to VAR2CSA and absence of PM}

To test whether lack of particular $\mathrm{Ab}$ responses to VAR2CSA were associated with absence of PM, a logistic regression models was used (Table 3). The logistic regression models showed no increase with protection from absence of PM at delivery with high Ab levels to individual domains, thus confirming results in Table 2 . However, higher avidity Ab to FV2 were significantly associated with the absence of PM ( $\mathrm{p}=0.0033)$; i.e. a $5 \%$ increase in Ab avidity to FV2 was associated with a $15 \%$ increase in the likelihood of a woman being protected from PM $(95 \%$ CI 1.01, 1.05) for the unadjusted model (Table 3). Ab avidity to FV2 remained significantly associated with protection from PM when logistic regression models were adjusted for gravidity only or gravidity and age (Table 3).

\section{Discussion}

In this study, Cameroonian multigravidae in a low malaria transmission area acquired high $\mathrm{Ab}$ levels to both the N-terminal and C-terminal regions of VAR2CSA by the third pregnancy. The primary difference between multigravidae who were PM-negative was that they had fine-tuned their immune responses through affinity maturation as reflected by increases in the proportion of high avidity Ab to FV2 ( $\mathrm{p}=0.003)$ (Table 3$)$. Examination of 
Table 2 Antibody response to malaria antigens in pregnant women at delivery

\begin{tabular}{|c|c|c|c|}
\hline Antibody response $^{a}$ & $\begin{array}{l}\text { PM positive } \\
(\mathrm{n}=96)\end{array}$ & $\begin{array}{l}\text { PM negative } \\
(\mathrm{n}=324)\end{array}$ & p value \\
\hline DBL1 3D7 (Pichia pastoris) (mean \pm SD) & $4832 \pm 4487$ & $4569 \pm 4031$ & 0.61 \\
\hline DBL1 7G8 (Pichia pastoris) (mean \pm SD) & $6479 \pm 4095$ & $6558 \pm 4237$ & 0.70 \\
\hline $\mathrm{DBL} 1+2 \mathrm{FCR3}$ (Baculovirus) (mean $\pm \mathrm{SD}$ ) & $8582 \pm 6649$ & $7901 \pm 5996$ & 0.37 \\
\hline DBL2 FCR3 (Baculovirus) (mean \pm SD) & $2416 \pm 2709$ & $2622 \pm 3041$ & 0.53 \\
\hline DBL3 FCR3 (Baculovirus) (mean \pm SD) & $6917 \pm 5052$ & $7524 \pm 5675$ & 0.32 \\
\hline DBL3 7 G8 (Pichia pastoris) (mean \pm SD) & $9940 \pm 6437$ & $10,986 \pm 6661$ & 0.17 \\
\hline DBL3 FCR3 (Escherichia coli) (mean \pm SD) & $7535 \pm 5542$ & $8171 \pm 5977$ & 0.34 \\
\hline DBL4 FCR3 (Baculovirus) (mean \pm SD) & $7966 \pm 4666$ & $8130 \pm 5481$ & 0.78 \\
\hline DBL4 7 G8 (Pichia pastoris) (mean \pm SD) & $4476 \pm 4130$ & $4594 \pm 4510$ & 0.73 \\
\hline DBL4 FCR3/IT4 (Pichia pastoris) (mean \pm SD) & $6274 \pm 4333$ & $5296 \pm 4428$ & 0.056 \\
\hline DBL5 FCR3 (Baculovirus) (mean \pm SD) & $15,749 \pm 6804$ & $15,270 \pm 6213$ & 0.54 \\
\hline DBL5 3D7 (Pichia pastoris) (mean \pm SD) & $13,237 \pm 5857$ & $12,124 \pm 5727$ & 0.11 \\
\hline DBL5 7 G8 (Pichia pastoris) (mean \pm SD) & $13,043 \pm 5563$ & $12,403 \pm 5524$ & 0.33 \\
\hline DBL6 FCR3 (Baculovirus) (mean \pm SD) & $7,655 \pm 5726$ & $8078 \pm 6287$ & 0.54 \\
\hline DBL6 7 G8 (Pichia pastoris) (mean \pm SD) & $7,293 \pm 4953$ & $7459 \pm 4486$ & 0.77 \\
\hline DBL6 FCR3/IT4 (Pichia pastoris) (mean \pm SD) & $4,364 \pm 3473$ & $4609 \pm 3577$ & 0.55 \\
\hline FV2 FCR3 (Baculovirus) (mean \pm SD) & $11,280 \pm 5293$ & $11,475 \pm 5654$ & 0.76 \\
\hline Per cent high avidity to FV2 (mean \pm SD) & $29.3 \pm 12.4$ & $33.7 \pm 12.6$ & 0.0030 \\
\hline Per cent of women with $\geq 35 \%$ high avidity Ab (\%) & $37.5 \%$ & $46.9 \%$ & 0.11 \\
\hline Number of domains recognized out of 6 (mean \pm SD) & $4.2 \pm 1.3$ & $4.1 \pm 1.3$ & 0.65 \\
\hline Number of variants recognized out of 15 (mean \pm SD) & $9.3 \pm 3.7$ & $9.1 \pm 3.6$ & 0.67 \\
\hline $\mathrm{CSP}($ mean $\pm \mathrm{SD})$ & $920 \pm 1030$ & $974 \pm 1066$ & 0.66 \\
\hline RESA (mean $\pm S D$ ) & $1033 \pm 2094$ & $1047 \pm 1861$ & 0.96 \\
\hline AMA-1 3D7 (mean \pm SD) & $14,814 \pm 4477$ & $12,004 \pm 5083$ & $<0.0001$ \\
\hline MSP1 3D7 (mean \pm SD) & $9051 \pm 6976$ & $7360 \pm 6246$ & 0.035 \\
\hline MSP2 3D7 (mean \pm SD) & $16,224 \pm 8444$ & $13,224 \pm 8658$ & 0.0028 \\
\hline
\end{tabular}

a For the comparison between PM+ and PM - women in antibody response, two sample t tests for continuous variables and Chi square tests for categorical variables were used

$p$ values that were less than 0.05 were designated in italics

Ab levels to DBL domains and FV2, as well as breadth of response (number of domains and variants recognized) in multigravidae using a logistic regression model revealed that increase in Ab levels to DBL domains or FV2 was not associated with reduced likelihood of PM. On the other hand, an increase in the proportion of high avidity Ab to FV2 was significantly associated with reduced likelihood of PM at delivery in multigravidae (Table 3). This association continued to be significant even after adjusting model for gravidity and age.

Protection from PM is mediated by Ab to VAR2CSA. However, the exact mechanism of protection has not been elucidated. Currently available effector mechanisms of IgG to VAR2CSA include inhibition of binding [6, 8, 11, 32] and opsonic phagocytosis [5, 15, 17]. Another essential characteristic of Ab to VAR2CSA is Ab affinity. While affinity describes the interaction between a single $\mathrm{Ab}$ combining site and cognate antigenic determinant, avidity describes the overall strength of attachment of heterogeneous polyclonal IgG to multiple determinants on complex antigens. Data from previous study demonstrated that in high malaria transmission settings, the proportion of high avidity Ab to FV2 was significantly higher in PM- compared to PM+ women [19]. In the current study, results indicate that multigravidae living in low malaria transmission area, a $5 \%$ difference in avidity of $\mathrm{Ab}$ to FV2 is associated with $15 \%$ lower likelihood of PM in multigravidae (Table 3), while prevalence of women with PM is reduced with higher number of pregnancies.

Affinity maturation is an antigen-driven process [22, 33 and leads to enhanced $B$ cells that progressively produce $\mathrm{Ab}$ with higher affinities and faster on-rates [22, 34]. A minority of $B$ cells with affinity-enhancing mutations differentiate into long-lived $\mathrm{Ab}$-secreting plasma cells and memory B cells. In diverse B cell receptor affinity population, B cells with high affinity B cell receptor have competitive advantage in binding to cognate antigen 
Table 3 Association between antibodies to VAR2CSA with absence of placental malaria in 420 women

\begin{tabular}{|c|c|c|c|c|c|c|c|}
\hline & \multirow[t]{2}{*}{ Increment unit } & \multicolumn{2}{|l|}{ Unadjusted } & \multicolumn{2}{|l|}{ Adjusted $^{a}$} & \multicolumn{2}{|l|}{ Adjusted $^{b}$} \\
\hline & & OR (95 \% Cl) & p value & OR $(95 \% \mathrm{Cl})$ & p value & OR $(95 \% \mathrm{Cl})$ & $\mathrm{p}$ value \\
\hline \multicolumn{8}{|l|}{ Immune responses to VAR2CSA } \\
\hline DBL1 3D7 (Pichia pastoris) & 5147.5 & $0.93(0.70,1.22)$ & 0.59 & $0.90(0.67,1.20)$ & 0.47 & $0.91(0.68,1.21)$ & 0.51 \\
\hline DBL1 7G8 (Pichia pastoris) & 5543.8 & $0.94(0.70,1.27)$ & 0.70 & $0.95(0.70,1.28)$ & 0.73 & $0.95(0.70,1.29)$ & 0.75 \\
\hline DBL1 + 2 FCR3 (Baculovirus) & 9077.3 & $0.85(0.61,1.19)$ & 0.35 & $0.87(0.62,1.22)$ & 0.43 & $0.88(0.63,1.23)$ & 0.46 \\
\hline DBL2 FCR3 (Baculovirus) & 2087.0 & $1.05(0.89,1.25)$ & 0.55 & $1.07(0.90,1.27)$ & 0.48 & $1.08(0.90,1.29)$ & 0.40 \\
\hline DBL3 FCR3 (Baculovirus) & 8125.0 & $1.18(0.84,1.67)$ & 0.89 & $1.22(0.86,1.72)$ & 0.27 & $1.23(0.87,1.75)$ & 0.25 \\
\hline DBL3 7G8 (Pichia pastoris) & $11,573.0$ & $1.33(0.88,1.99)$ & 0.18 & $1.28(0.85,1.93)$ & 0.24 & $1.27(0.85,1.92)$ & 0.25 \\
\hline DBL3 FCR3 (Escherichia coli) & 9346.8 & $1.19(0.82,1.73)$ & 0.36 & $1.16(0.80,1.68)$ & 0.44 & $1.15(0.79,1.67)$ & 0.46 \\
\hline DBL4 FCR3 (Baculovirus) & 7023.0 & $1.04(0.77,1.41)$ & 0.79 & $1.06(0.78,1.44)$ & 0.73 & $1.07(0.78,1.46)$ & 0.68 \\
\hline DBL4 7G8 (Pichia pastoris) & 5244.3 & $0.96(0.73,1.25)$ & 0.74 & $0.95(0.72,1.24)$ & 0.69 & $0.94(0.72,1.24)$ & 0.68 \\
\hline DBL4 FCR3/IT4 (Pichia pastoris) & 5088.3 & $0.79(0.61,1.01)$ & 0.059 & $0.80(0.62,1.03)$ & 0.080 & $0.80(0.63,1.03)$ & 0.086 \\
\hline DBL5 FCR3 (Baculovirus) & $11,083.0$ & $0.88(0.59,1.31)$ & 0.52 & $0.88(0.59,1.32)$ & 0.54 & $0.87(0.58,1.30)$ & 0.49 \\
\hline DBL5 3D7 (Pichia pastoris) & 9502.3 & $0.72(0.49,1.06)$ & 0.098 & $0.72(0.49,1.06)$ & 0.097 & $0.71(0.48,1.04)$ & 0.079 \\
\hline DBL5 7 G8 (Pichia pastoris) & 9335.5 & $0.82(0.55,1.21)$ & 0.32 & $0.81(0.55,1.21)$ & 0.31 & $0.80(0.54,1.19)$ & 0.27 \\
\hline DBL6 FCR3 (Baculovirus) & 9534.0 & $1.11(0.78,1.60)$ & 0.56 & $1.09(0.76,1.56)$ & 0.66 & $1.09(0.76,1.56)$ & 0.66 \\
\hline DBL6 7G8 (Pichia pastoris) & 6554.5 & $1.05(0.76,1.46)$ & 0.76 & $1.08(0.78,1.50)$ & 0.66 & $1.05(0.76,1.47)$ & 0.76 \\
\hline DBL6 FCR3/IT4 (Pichia pastoris) & 3803.5 & $1.08(0.84,1.39)$ & 0.56 & $1.05(0.82,1.36)$ & 0.69 & $1.05(0.82,1.35)$ & 0.72 \\
\hline FV2 FCR3 (Baculovirus) & 9579.5 & $1.06(0.72,1.57)$ & 0.77 & $1.06(0.71,1.57)$ & 0.80 & $1.05(0.71,1.57)$ & 0.81 \\
\hline Per cent high avidity to FV2 & 1 & $1.03(1.01,1.05)$ & 0.0033 & $1.03(1.01,1.05)$ & 0.0079 & $1.03(1.01,1.04)$ & 0.010 \\
\hline $\begin{array}{l}\text { Per cent of women with } \geq 35 \% \text { high avidity Ab } \\
\text { to FV2 }\end{array}$ & 1 & $1.47(0.92,2.35)$ & 0.11 & $1.45(0.90,2.32)$ & 0.13 & $1.43(0.89,2.30)$ & 0.15 \\
\hline Number of domains recognized out of 6 & 1 & $0.96(0.81,1.14)$ & 0.65 & $0.96(0.81,1.15)$ & 0.67 & $0.96(0.81,1.15)$ & 0.68 \\
\hline Number of variants recognized out of 15 & 1 & $0.99(0.93,1.05)$ & 0.67 & $0.98(0.92,1.05)$ & 0.56 & $0.98(0.92,1.05)$ & 0.58 \\
\hline \multicolumn{8}{|c|}{ Immune responses to non-pregnancy associated antigens } \\
\hline CSP & 875.5 & $1.05(0.86,0.27)$ & 0.66 & $1.00(0.82,1.22)$ & 1.00 & $1.01(0.83,1.24)$ & 0.93 \\
\hline RESA & 512.5 & $1.02(0.94,1.07)$ & 0.95 & $1.00(0.94,1.06)$ & 0.90 & $1.00(0.94,1.06)$ & 0.90 \\
\hline AMA-1 3D7 & 7905.8 & $0.36(0.23,0.56)$ & $<0.0001$ & $0.37(0.24,0.58)$ & $<0.0001$ & $0.37(0.24,0.57)$ & $<0.0001$ \\
\hline MSP1 3D7 & $11,844.8$ & $0.62(0.41,0.94)$ & 0.025 & $0.60(0.39,0.92)$ & 0.019 & $0.61(0.40,0.94)$ & 0.025 \\
\hline MSP2 3D7 & $18,300.8$ & $0.47(0.29,0.78)$ & 0.0032 & $0.47(0.28,0.78)$ & 0.0034 & $0.47(0.28,0.78)$ & 0.0038 \\
\hline
\end{tabular}

The antibody levels (MFI) were rescaled by IQR (the difference between 75th and 25th percentiles)

$p$ values that were less than 0.05 were designated in italics

a Logistic regression models were adjusted for gravidity

b Logistic regression models were adjusted for gravidity and age

in an antigen-limiting environment. This view is in line with the findings that when immunogen is present in large doses the rate at which the affinity of serum $\mathrm{Ab}$ is increased is greatly reduced, since high levels of antigen on follicular dendritic cells reduce competitive advantage of high affinity B cell receptor [35].

$\mathrm{Ab}$ avidity data for VAR2CSA are not available for other countries. However, outside pregnancy, the notion that $\mathrm{Ab}$ quality is important for protection has been suggested [36], since similar IgG levels to $P$. falciparum were found in patients with severe and mild malaria [37]. High avidity Ab to an extract of $P$. falciparum schizonts (Amazonian parasite isolate) have been detected in clinically immune Senegalese adults [38]; high avidity Ab to blood stage antigen were observed among asymptomatic Brazilian adults [39]; affinity of Ab to AMA-1 and MSP2 increased with age and high affinity Ab to MSP2 were shown to prolong time to re-infection in Ugandan and Tanzanian children [40]. However, Ab avidity to AMA1, MSP1 and MSP3 were not associated with immunity to malaria among Kenyan [41] and Gambian children [42], possibly due to differences in techniques used and malaria transmission. In addition, $\mathrm{Ab}$ avidity can be important for mediating effector functions against placental-binding $P$. falciparum, such as inhibition of binding or opsonic phagocytosis. Humburger et al. 
demonstrated that protective nature of anti-malarial $\mathrm{Ab}$ could be reversed due to the low Ab avidity [43].

Of interest, Cameroonian pregnant women in this study had predominantly cytophilic IgG1 sub-class of Ab as reported previously [44]. IgG1 and IgG3 to variant surface antigen are associated with high opsonic phagocytosis activity of CSA-binding IE in Kenyan women; further, IgG1 and IgG3 levels to variant surface antigen on CSAbinding IE have strong positive correlation with phagocytic index [15]. In contrast, Guitard et al. found that VAR2CSA DBL5 in Senegalese pregnant women elicits high levels of both IgG1 and IgG3 [45].

In this study, several males living in Yaounde had $\mathrm{Ab}$ to VAR2CSA domains that were above cut-off (Additional file 1). This phenomena has been observed in previous studies conducted in rural Cameroonian villages with high malaria transmission (in press). It is likely that in individuals with high, chronic parasitemia, parasites switch to the VAR2CSA-expressing phenotype and induce an $\mathrm{Ab}$ response. For example, $\mathrm{Ab}$ levels to DBL5 have been reported in young Tanzanian children [46] with severe malaria and in more than $60 \%$ Colombian men and children [47]. Another possibility is that conserved epitopes between VAR2CSA and other Plasmodium falciparum Erythrocyte Membrane P1 family antigens exist. However, results from a study by Beeson et al., who used plasma from men and children living in Papua New Guinea, Kenya and Malawi in a cell surface binding assay using both VAR2CSA-expressing and nonVAR2CSA expressing IE [48], showed that IgG-binding was specific and did not represent $\mathrm{Ab}$ to subpopulations of non-CSA-binding IE. Further, sera from some nonpregnant individuals inhibited IE adhesion to CSA. Hence, it is likely that IgG observed in non-pregnant individuals are specific to VAR2CSA and are not due to the cross-reactivity with non-VAR2CSA surface antigens.

This study had several limitations. One key limitation was a cross-sectional nature of the original study and therefore absence of data on clinical and malaria exposure of the pregnant women throughout pregnancy. Thus, stratification of women into better-defined groups based on their clinical histories was not possible. Additional limitations include: absence of PM categorization according to Bulmer score and only one form of FV2 was available for testing. Finally, information on functional properties of Ab to VAR2CSA were not included in this analysis. Currently, studies on opsonic phagocytosis and inhibition of binding to CSA are being carried out.

Finally, protection from PM depends on acquisition of $\mathrm{Ab}$ to VAR2CSA. It is not clear whether protection could be mediated by high titres and affinity maturation to a single conserved VAR2CSA epitope, perhaps within the CSA-binding site, during repeat infections or acquisition of a broad range of IgG to many variants (genotypes) of VAR2CSA, that is, protection would require $A b$ to 'enough' variants. Since in multigravidae total antiVAR2CSA IgG levels did not increase with increasing gravidities, it is possible that CSA-binding parasites express 'decoy' conserved immunodominant epitopes, which elicit high IgG titres that are not associated with absence of PM. Multigravidae in the present study continued to have PM even though they had high IgG titers to various VAR2CSA domains, e.g., DBL3, DBL5 and DBL6. In addition, no single VAR2CSA domain tested was associated with absence of PM in this study. If protection from $\mathrm{PM}$ is mediated by a single conserved sequence or domain of VAR2CSA, Ab boosting to that sequence might be difficult to detect using the method employed in this study. Since individual recombinant DBL domains used in the study were "removed from the molecule," it is also possible the conformational epitopes created by different domains in the intact antigen are critical for inducing protection. Overall, data reported in this study support the conclusion that Ab avidity to FV2 plays a role in immunity to PM.

$\mathrm{Ab}$ avidity is an important determinant of successful vaccination against infections such as H1N1 [49], Hib Haemophiluys influenzae type B [50] and Bordetella pertussis [51]. In addition, recent studies in African infants who received RTS,S/AS01E in phase 2 trial demonstrated that "change in avidity following second and third RTS,S/ AS01E injection was associated with a $54 \%$ likelihood reduction of getting malaria" [52]. Whether high avidity $\mathrm{Ab}$ to VAR2CSA can be induced by vaccination remains unknown.

\section{Conclusion}

Results from this study suggest that high avidity $\mathrm{Ab}$ to VAR2CSA may play a key role in immunity to PM. It is conceivable that Ab avidity to FV2 is an important surrogate marker for assessing VAR2CSA vaccine efficacy.

\section{Additional files}

Additional file 1. Cut-off values for DBL domains and FV2.

Additional file 2. Testing mouse anti-human monoclonal antibodies for cross-reactivity (median fluorescent intensities tabulated).

Additional file 3. IgG sub-class levels to the full-length VAR2CSA. IgG sub-class levels to FV2 FCR3 were measured in randomly selected 20 $\mathrm{PM}+$ and $20 \mathrm{PM}$-negative women. Median and interquartile ranges plotted.

\section{Abbreviations}

Ab: antibodies; AID: activation-induced cytidine deaminase; BSA: bovine serum albumin; CSA: chondroitin sulfate A; DBL: VAR2CSA Duffy binding-like domains; FV2: full-length VAR2CSA; HIV: human immunodeficiency virus; IE: infected erythrocytes; IgG: immunoglobulin G; IPT: intermittent preventive treatment; MAP: multi-analyte platform assay; MFI: median fluorescent 
intensity; PBS: phosphate buffered saline; PCV: packed cell volume; PM: placental malaria; PM+: placental malaria positive; PM: placental malaria negative.

\section{Authors' contributions}

$A B$ helped design the study, carried out immunological assays, analysed and interpreted the data and wrote manuscript; RF, AW and JJC analysed the data interpreted the results and assisted with manuscript preparation; GS and CE collected and processed samples, determined parasitaemia and provided haematological results; AS provided recombinant proteins and helpful comments in the Discussion; RJIL was responsible for all aspects of the clinical components of the study; RGFL oversaw all aspects of the study in Cameroon and participated in manuscript preparation; DWT designed the study, oversaw data collection, interpreted results, and participated in manuscript preparation. All authors read and approved the final manuscript.

\section{Author details}

1 Department of Tropical Medicine, Medical Microbiology and Pharmacology, John A Burns School of Medicine, University of Hawaii at Manoa, 651 Ilalo Street, BSB 320, Honolulu, HI 96813, USA. ${ }^{2}$ Faculty of Medicine and Biomedical Research, Biotechnology Centre, University of Yaounde 1, Yaounde, Cameroon. ${ }^{3}$ Department of Immunology and Microbiology, Centre for Medical Parasitology, University of Copenhagen, Copenhagen, Denmark.

\section{Acknowledgements}

We acknowledge the support of the entire Malaria Research Team at the Biotechnology Center, University of Yaoundé I, Cameroon, for their outstanding work, and the women and their families who participated in the studies. We acknowledge Dr. J Smith (Seattle Biomedical Research Institute), C Long and K Singh (NIAID) for providing recombinant proteins used in this study. Finally, we thank A Kayatani (WRAIR, US Army Forces) for providing expertise with the avidity and IgG sub-class assay. The work was supported by grants from NIAID, $\mathrm{NIH}$, UO1AI43888 and RO1Al071160 (DWT, RGFL), R21Al105286-01 (DWT, JJC), NIMHD U54MD007584 and NIH G12MD007601 (RF, AW, JJC). AB was supported by University of Hawaii John A Burns School of Medicine Dean's scholarship, University of Hawaii Manoa grant, University of Hawaii Tropical Medicine Departmental scholarship and NIH Fogarty Research Training Grant \#R25 TW009345 awarded to the Northern Pacific Global Health Fellows. The content is solely the responsibility of the authors and does not necessarily represent the official views of the $\mathrm{NIH}$.

\section{Competing interests}

The authors declare that they have no competing interests.

Received: 22 September 2015 Accepted: 26 November 2015 Published online: 01 December 2015

\section{References}

1. Brabin BJ, Premji Z, Verhoeff F. An analysis of anemia and child mortality. J Nutr. 2001;131:636S-45S (discussion 646S-648S)

2. Bockhorst J, Lu F, Janes JH, Keebler J, Gamain B, Awadalla P, et al. Structural polymorphism and diversifying selection on the pregnancy malaria vaccine candidate VAR2CSA. Mol Biochem Parasitol. 2007;155:103-12.

3. Barfod L, Dobrilovic T, Magistrado P, Khunrae P, Viwami F, Bruun J, et al. Chondroitin sulfate A-adhering Plasmodium falciparum-infected erythrocytes express functionally important antibody epitopes shared by multiple variants. J Immunol. 2010;185:7553-61.

4. Khunrae P, Dahlback M, Nielsen MA, Andersen G, Ditlev SB, Resende M, et al. Full-length recombinant Plasmodium falciparum VAR2CSA binds specifically to CSPG and induces potent parasite adhesion-blocking antibodies. J Mol Biol. 2010;397:826-34.

5. Feng G, Aitken E, Yosaatmadja F, Kalilani L, Meshnick SR, Jaworowski A, et al. Antibodies to variant surface antigens of Plasmodium falciparum-infected erythrocytes are associated with protection from treatment failure and the development of anemia in pregnancy. J Infect Dis. 2009;200:299-306.

6. O'Neil-Dunne I, Achur RN, Agbor-Enoh ST, Valiyaveettil M, Naik RS, Ockenhouse CF, et al. Gravidity-dependent production of antibodies that inhibit binding of Plasmodium falciparum-infected erythrocytes to placental chondroitin sulfate proteoglycan during pregnancy. Infect Immun. 2001;69:7487-92.

7. Staalsoe T, Megnekou R, Fievet N, Ricke CH, Zornig HD, Leke R, et al. Acquisition and decay of antibodies to pregnancy-associated variant antigens on the surface of Plasmodium falciparum-infected erythrocytes that protect against placental parasitemia. J Infect Dis. 2001;184:618-26.

8. Duffy PE, Fried M. Antibodies that inhibit Plasmodium falciparum adhesion to chondroitin sulfate $A$ are associated with increased birth weight and the gestational age of newborns. Infect Immun. 2003;71:6620-3.

9. Nielsen MA, Resende M, de Jongh WA, Ditlev SB, Mordmüller B, Houard $S$, et al. The influence of sub-unit composition and expression system on the functional antibody response in the development of a VAR2CSA based Plasmodium falciparum placental malaria vaccine. PLoS One. 2015;10:e0135406.

10. Clausen TM, Christoffersen S, Dahlback M, Langkilde AE, Jensen KE, Resende $M$, et al. Structural and functional insight into how the Plasmodium falciparum VAR2CSA protein mediates binding to chondroitin sulfate A in placental malaria. J Biol Chem. 2012;287:23332-45.

11. Ndam NT, Denoeud-Ndam L, Doritchamou J, Viwami F, Salanti A, Nielsen MA, et al. Protective antibodies against placental malaria and poor outcomes during pregnancy, Benin. Emerg Infect Dis. 2015;21:813-23.

12. Dechavanne S, Srivastava A, Gangnard S, Nunes-Silva S, Dechavanne C, Fievet N, et al. Parity-dependent recognition of DBL1X-3X suggests an important role of the VAR2CSA high-affinity CSA-binding region in the development of the humoral response against placental malaria. Infect Immun. 2015;83:2466-74.

13. Saveria T, Oleinikov AV, Wiliamson K, Chaturvedi R, Lograsso J, Keitany GJ, et al. Antibodies to Escherichia coli-expressed C-terminal domains of Plasmodium falciparum variant surface antigen 2-chondroitin sulfate $A$ (VAR2CSA) inhibit binding of CSA-adherent parasites to placental tissue. Infect Immun. 2013;81:1031-9.

14. Brolin KJ, Persson KE, Wahlgren M, Rogerson SJ, Chen Q. Differential recognition of P. falciparum VAR2CSA domains by naturally acquired antibodies in pregnant women from a malaria endemic area. PLoS One. 2010;5:e9230.

15. Keen J, Serghides L, Ayi K, Patel SN, Ayisi J, van Eijk A, et al. HIV impairs opsonic phagocytic clearance of pregnancy-associated malaria parasites. PLos Med. 2007:4:e181.

16. Lambert LH, Bullock JL, Cook ST, Miura K, Garboczi DN, Diakite M, et al. Antigen reversal identifies targets of opsonizing IgGs against pregnancyassociated malaria. Infect Immun. 2014;82:4842-53.

17. Ataide R, Mwapasa V, Molyneux ME, Meshnick SR, Rogerson SJ. Antibodies that induce phagocytosis of malaria infected erythrocytes: effect of HIV infection and correlation with clinical outcomes. PLoS One. 2011;6:e22491.

18. Tutterrow YL, Avril M, Singh K, Long CA, Leke RJ, Sama G, et al. High levels of antibodies to multiple domains and strains of VAR2CSA correlate with the absence of placental malaria in Cameroonian women living in an area of high Plasmodium falciparum transmission. Infect Immun. 2012;80:1479-90.

19. Tutterrow YL, Salanti A, Avril M, Smith JD, Pagano IS, Ako S, et al. High avidity antibodies to full-length VAR2CSA correlate with absence of placental malaria. PLoS One. 2012;7:e40049.

20. Berek C, Berger A, Apel M. Maturation of the immune response in germinal centers. Cell. 1991;67:1121-9.

21. French DL, Laskov R, Scharff MD. The role of somatic hypermutation in the generation of antibody diversity. Science. 1989;244:1152-7.

22. Schallert N, Pihlgren M, Kovarik J, Roduit C, Tougne C, Bozzotti P, et al. Generation of adult-like antibody avidity profiles after early-life immunization with protein vaccines. Eur J Immunol. 2002;32:752-60.

23. Dellicour S, Tatem AJ, Guerra CA, Snow RW, ter Kuile FO. Quantifying the number of pregnancies at risk of malaria in 2007: a demographic study. PLos Med. 2010;7:e1000221.

24. Tako EA, Zhou A, Lohoue J, Leke R, Taylor DW, Leke RF. Risk factors for placental malaria and its effect on pregnancy outcome in Yaounde, Cameroon. Am J Trop Med Hyg. 2005;72:236-42.

25. Suguitan AL Jr, Cadigan TJ, Nguyen TA, Zhou A, Leke RJ, Metenou S, et al. Malaria-associated cytokine changes in the placenta of women with pre-term deliveries in Yaounde, Cameroon. Am J Trop Med Hyg. 2003;69:574-81. 
26. Louis JP, Trebucq A, Gelas H, Fondjo E, Manga L, Toto JC, et al. Malaria in Yaounde (Cameroon). Cost and antivectorial control at the family level (in French). Bull Soc Pathol Exot. 1992;85:26-30.

27. Manga L, Traore O, Cot M, Mooh E, Carnevale P. Malaria in the village of Yaounde (Cameroon). 3. Parasitological study in 2 central districts (in French). Bull Soc Pathol Exot. 1993;86:56-61.

28. World Health Organization. Report on the global HIV/AIDS epidemic, 2002. http://www.who.int/hiv/pub/epidemiology/hiv_aids_2001.xls.

29. Avril M, Kulasekara BR, Gose SO, Rowe C, Dahlback M, Duffy PE, et al. Evidence for globally shared, cross-reacting polymorphic epitopes in the pregnancy-associated malaria vaccine candidate VAR2CSA. Infect Immun. 2008;76:1791-800.

30. Robson KJH, Walliker D, Creasey A, JMcBride J, Beale G, Wilson RJM. Crosscontamination of Plasmodium cultures. Parasitol Today. 1992;8:38-9.

31. Fouda GG, Leke RF, Long C, Druilhe P, Zhou A, Taylor DW, et al. Multiplex assay for simultaneous measurement of antibodies to multiple Plasmodium falciparum antigens. Clin Vaccine Immunol. 2006;13:1307-13.

32. Fried M, Nosten F, Brockman A, Brabin BJ, Duffy PE. Maternal antibodies block malaria. Nature. 1998;395:851-2.

33. McHeyzer-Williams MG, McLean MJ, Lalor PA, Nossal GJ. Antigen-driven B cell differentiation in vivo. J Exp Med. 1993;178:295-307.

34. Foote J, Milstein C. Kinetic maturation of an immune response. Nature. 1991;352:530-2

35. Eisen HN, Siskind GW. Variations in affinities of antibodies during the immune response. Biochemistry. 1964;3:996-1008.

36. Bouharoun-Tayoun H, Druilhe P. Antibodies in falciparum malaria: what matters most, quantity or quality? Mem Inst Oswaldo Cruz. 1992;87(Suppl 3):229-34.

37. Erunkulu OA, Hill AV, Kwiatkowski DP, Todd JE, lqbal J, Berzins K, et al. Severe malaria in Gambian children is not due to lack of previous exposure to malaria. Clin Exp Immunol. 1992;89:296-300.

38. Ferreira MU, Kimura EA, De Souza JM, Katzin AM. The isotype composition and avidity of naturally acquired anti-Plasmodium falciparum antibodies: differential patterns in clinically immune Africans and Amazonian patients. Am J Trop Med Hyg. 1996:55:315-23.

39. Leoratti FM, Durlacher RR, Lacerda MV, Alecrim MG, Ferreira AW, Sanchez $M C$, et al. Pattern of humoral immune response to Plasmodium falciparum blood stages in individuals presenting different clinical expressions of malaria. Malar J. 2008;7:186.

40. Reddy SB, Anders RF, Beeson JG, Farnert A, Kironde F, Berenzon SK, et al. High affinity antibodies to Plasmodium falciparum merozoite antigens are associated with protection from malaria. PLoS One. 2012;7:e32242.

41. Ibison F, Olotu A, Muema DM, Mwacharo J, Ohuma E, Kimani D, et al. Lack of avidity maturation of merozoite antigen-specific antibodies with increasing exposure to Plasmodium falciparum amongst children and adults exposed to endemic malaria in Kenya. PLoS One. 2012;7:e52939.
42. Akpogheneta OJ, Dunyo S, Pinder M, Conway DJ. Boosting antibody responses to Plasmodium falciparum merozoite antigens in children with highly seasonal exposure to infection. Parasite Immunol. 2010;32:296-304

43. Hamburger J, Kreier JP. Interaction between protective antibodies and malaria parasites (Plasmodium berghei): involvement of low avidity antibodies. Tropenmed Parasitol. 1976;27:385-90.

44. Megnekou R, Staalsoe T, Taylor DW, Leke R, Hviid L. Effects of pregnancy and intensity of Plasmodium falciparum transmission on immunoglobulin $\mathrm{G}$ subclass responses to variant surface antigens. Infect Immun. 2005:73:4112-8.

45. Guitard J, Cottrell G, Magnouha NM, Salanti A, Li T, Sow S, et al. Differential evolution of anti-VAR2CSA- IgG3 in primigravidae and multigravidae pregnant women infected by Plasmodium falciparum. Malar J. 2008;7:10.

46. Oleinikov AV, Voronkova WV, Frye IT, Amos E, Morrison R, Fried M, et al. A plasma survey using 38 PfEMP1 domains reveals frequent recognition of the Plasmodium falciparum antigen VAR2CSA among young Tanzanian children. PLoS One. 2012;7:e31011.

47. Gnidehou S, Doritchamou J, Arango EM, Cabrera A, Arroyo MI, Kain KC, et al. Functional antibodies against VAR2CSA in nonpregnant populations from colombia exposed to Plasmodium falciparum and Plasmodium vivax. Infect Immun. 2014:82:2565-73.

48. Beeson JG, Ndungu F, Persson KE, Chesson JM, Kelly GL, Uyoga S, et al. Antibodies among men and children to placental-binding Plasmodium falciparum-infected erythrocytes that express var2csa. Am J Trop Med Hyg. 2007;77:22-8.

49. Khurana S, Verma N, Talaat KR, Karron RA, Golding H. Immune response following $\mathrm{H} 1 \mathrm{~N} 1 \mathrm{pdm} 09$ vaccination: differences in antibody repertoire and avidity in young adults and elderly populations stratified by age and gender. J Infect Dis. 2012;205:610-20.

50. Denoel PA, Goldblatt D, de Vleeschauwer I, Jacquet JM, Pichichero ME, Poolman JT. Quality of the Haemophilus influenzae type b (Hib) antibody response induced by diphtheria-tetanus-acellular pertussis/Hib combination vaccines. Clin Vaccine Immunol. 2007;14:1362-9.

51. Prelog M, Almanzar G, Rieber N, Ottensmeier B, Zlamy M, Liese J. Differences of IgG antibody avidity after an acellular pertussis (aP) booster in adolescents after a whole cell (WCP) or aP primary vaccination. Vaccine. 2013:31:387-93.

52. Ajua A, Lell B, Agnandji ST, Asante KP, Owusu-Agyei S, Mwangoka G, et al. The effect of immunization schedule with the malaria vaccine candidate RTS, S/AS01E on protective efficacy and anti-circumsporozoite protein antibody avidity in African infants. Malar J. 2015;14:72.

\section{Submit your next manuscript to BioMed Central and we will help you at every step:}

- We accept pre-submission inquiries

- Our selector tool helps you to find the most relevant journal

- We provide round the clock customer support

- Convenient online submission

- Thorough peer review

- Inclusion in PubMed and all major indexing services

- Maximum visibility for your research

Submit your manuscript at www.biomedcentral.com/submit
() Biomed Central 